\title{
La souscription nationale pour sauvegarder les manuscrits de Robespierre : introspection historique d'une initiative citoyenne et militante
}

The national subscription to protect Robespierre's manuscripts : historical introspection of an militant and civic initiative

\section{Serge Aberdam et Cyril Triolaire}

\section{(2) OpenEdition Journals}

\section{Édition électronique}

URL : https://journals.openedition.org/ahrf/12674

DOI : 10.4000/ahrf.12674

ISSN : $1952-403 X$

\section{Éditeur :}

Armand Colin, Société des études robespierristes

\section{Édition imprimée}

Date de publication : 1 mars 2013

Pagination : 9-38

ISBN : 978-2-200-92824-7

ISSN : 0003-4436

\section{Référence électronique}

Serge Aberdam et Cyril Triolaire, «La souscription nationale pour sauvegarder les manuscrits de Robespierre : introspection historique d'une initiative citoyenne et militante », Annales historiques de la Révolution française [En ligne], 371 | janvier-mars 2013, mis en ligne le 01 mars 2016, consulté le 01 juillet 2021. URL : http://journals.openedition.org/ahrf/12674; DOI : https://doi.org/10.4000/ahrf. 12674 


\title{
LA SOUSCRIPTION NATIONALE POUR SAUVEGARDER LES MANUSCRITS DE ROBESPIERRE : INTROSPECTION HISTORIQUE D'UNE INITIATIVE CITOYENNE ET MILITANTE
}

\author{
Serge ABERDAM et Cyril TRIOLAIRE
}

\begin{abstract}
Fin avril 2011, la Société des études robespierristes apprend la mise aux enchères, chez Sotheby's, à Paris, de manuscrits inédits de Robespierre et de Le Bas. À l'émotion, partagée aussi à l'Institut d'histoire de la Révolution française et par de nombreuses associations amies, succède bientôt la réaction. Début mai, la Société des études robespierristes lance une souscription nationale inédite visant à récolter un maximum de fonds pour préserver dans les collections publiques les précieux brouillons de l'Incorruptible, ou au moins essayer de peser sur les institutions de conservation pour les inciter à agir. La souscription est un succès, appuyée à l'Assemblée nationale et au Sénat, largement relayée par les médias ; bientôt l'État préempte puis rachète les deux lots mis en vente. Durant plusieurs semaines, des courriers, accompagnés de dons, affluent de tout le pays ; émus et indignés, ils sont plus de mille à participer à cette souscription et à ainsi participer directement à cette opération citoyenne de sauvegarde de ce patrimoine national. Deux ans après, il est temps de revenir sur ce mouvement sans précédent, d'en saisir la dynamique, de dresser un portait de groupe de ces donateurs, de mesurer et de comprendre leur geste, sans lequel il va sans dire que ces manuscrits n'auraient très vraisemblablement pas été préservés dans les collections des Archives nationales.
\end{abstract}

Mots-clés : Société des études robespierristes, Révolution française, Robespierre, patrimoine national, manuscrits, vente aux enchères, souscription publique. 
La souscription pour sauver les manuscrits de Robespierre et de Lebas, mis en vente chez Sotheby's à Paris, a été lancée en mai 2011 par la Société des études robespierristes puis, de fait, s'est prolongée dans l'été. Le 5 novembre suivant, lorsque les donateurs et amis se réunissent à Ivry-sur-Seine pour célébrer l'entrée des manuscrits sauvegardés dans les collections publiques françaises, ils sont encore étonnés de leur propre succès. Aujourd'hui, il est certainement trop tôt pour entrer dans tous les détails de cette histoire, qui supposerait de s'appuyer sur la masse de messages alors échangés en tous sens. Il est déjà néanmoins possible de s'intéresser à cette initiative originale et à la mobilisation qu'elle a enclenchée, la première même dans son genre.

Dans les derniers jours d'avril 2011, lorsque la nouvelle de la mise en vente des papiers est délibérément répandue par l'organisme vendeur, le premier réflexe est de considérer que les Archives nationales vont au minimum faire connaître leur opposition à la sortie du territoire de ces documents exceptionnels, imposer leur autorité et aboutir à un compromis avant la vente. Si les indications contraires ne sont d'abord pas prises au sérieux, les fins de non recevoir, adressées poliment par les Archives nationales puis la Bibliothèque nationale de France à une première demande formulée par Philippe Bourdin, alertent rapidement les membres du bureau de la SER. Au moment où toute l'étendue du problème apparaît, le 4 mai, Pierre Serna, directeur de l'Institut d'histoire de la Révolution française, publie dans Le Monde une tribune appelant littéralement à « sauver Robespierre $»^{1}$. Moins de deux jours plus tard, les membres du bureau de la SER et son nouveau président Michel Biard décident de lancer un appel à souscription publique nationale, discuté déjà depuis près d'une semaine. Un premier temps fort s'inscrit alors dans les tâches quotidiennes du secrétariat de la SER. Ce secrétariat qu'on dit « général » est, plus modestement, derrière le président, un exécutif du conseil d'administration chargé au fond de garder en mémoire et d'essayer de mettre en musique les orientations votées. Dans ce cadre, la réalité de la mise en vente des papiers en question, la présence d'opérateurs décidés à faire monter les prix et l'implication initialement faible des pouvoirs publics provoquent notre indignation, le sentiment d'avoir un rôle à jouer, une mission d'intérêt public à remplir. 


\section{La Société des études robespierristes face à son avenir}

Immédiatement, le bureau est confronté à deux difficultés internes à la SER : son rapport au public et son rapport à l'argent. Côté public, quoi de plus banal qu'une association, une société savante bien vivante qui est également, selon la formule chère à Claude Mazauric, une société de pensée, mais qui a évolué au fil du temps ? Depuis plusieurs décennies, on voit s'affirmer le caractère académique de la Société avec son organe, les Annales historiques de la Révolution française, devenue la seule revue universitaire internationale qui soit consacrée à la Révolution française. Pareillement, on ne peut que constater l'usure insensible du collectif que forment les membres de la Société, évidemment accentuée par l'inévitable vieillissement. Les exposés et débats lors du colloque consacré en 2008 à son centième anniversaire étaient sans brutalité, mais sans indulgence. La SER est de composition de plus en plus universitaire, non seulement dans son recrutement mais aussi par les articles que publie sa revue. Les critères «d'excellence » imposés au monde de l'enseignement et de la recherche font qu'il est certainement plus difficile de nos jours de publier dans les Annales historiques de la Révolution française pour un historien exerçant dans l'enseignement secondaire ou primaire que cela ne l'était jadis pour, disons, un Maurice Dommanget, instituteur et (ô combien !) grand historien. L'équation peut être encore pire s'il s'agit d'un amateur, même très qualifié, ce qui n'est pas sans poser problème à notre époque de retraités très actifs. Le recrutement de la Société s'en ressent, et ce d'autant plus que les revues régionales et l'érudition dite locale ont de leur côté repris de la vigueur : la séparation qui s'aggrave ainsi est dommageable à tous points de vue, en particulier parce qu'elle diminue les nécessaires échanges, le nécessaire brassage. Depuis une dizaine d'années, ces questions sont débattues ouvertement et librement, avec des conséquences réelles quoiqu'encore limitées. La SER a ainsi pu mettre en place un prix de thèse, le prix Albert Mathiez, mais aussi organiser des chantiers mobilisateurs, comme le réseau qui travaille sur les comités de surveillance, les rencontres de juin 2010 sur les chantiers actuels de la recherche, ou encore celles de mars 2011 sur l'enseignement de la Révolution française en collège et lycée ; initiatives qui, toutes, visaient à élargir le public concerné.

Côté argent, quoi de plus banal à ce qu'une association comme la nôtre se débatte depuis des décennies dans ses comptes annuels et leur vérification, dans l'équilibrage des finances de sa revue et la pérennité de ses ressources? Les membres de nos conseils d'administration successifs ont 
dû faire face aux problèmes de révision, de fabrication, de tirage, de prix, de stockage, de gestion des droits. Une véritable saga en mode mineur qui ne trouvait son vrai cadre, justement, que dans notre qualité de bénévoles, dans le réel rayonnement de notre société et dans son solide bilan sur plus d'un siècle. On discerne cependant ici un rapport un peu difficile aux valeurs monétaires qui ne serait qu'un détail mineur s'il ne s'y ajoutait une autre caractéristique. En 1935, notre fondateur Albert Mathiez avait remporté de façon posthume une victoire d'importance en faisant reconnaître par décret notre société comme d'Utilité publique. Malheureusement, décédé en 1932, il n'était plus présent pour utiliser l'outil dont il avait demandé à disposer et qui permettait, cas rare à l'époque, d'importantes facilités en matière de dons, de legs et de fiscalité. Nul évidemment ne sait comment Mathiez aurait utilisé notre statut d'Utilité publique dans la préparation du cent-cinquantenaire prévu pour 1939, lui qui, quelques décennies plus tôt, avait si vertement critiqué chez Alphonse Aulard ce qu'il ressentait comme de l'affairisme. Reste que, au moins depuis 1945, les utilisations de notre statut d'Utilité publique pour recueillir des ressources, avec les déductions fiscales correspondantes pour les donateurs, sont restées très marginales au sein de la SER. De fait, celle-ci n'a-t-elle guère utilisé ce statut longtemps exceptionnel et avec lequel la masse des associations « loi de $1901 »$ n'a eu de cesse d'obtenir des rapprochements, encore en cours.

L'attitude de notre société était peut-être vertueuse mais aussi, certainement, paralysante. Il n'est que de suivre ce qu'ont pu faire du même outil associatif quelques sociétés sœurs ou amies pour évaluer ce que nous n'avons jamais pu rassembler comme moyens et donc ce qui n'a pu exister en matière de financement des recherches, d'aide à la publication, de réédition, de traduction, de conservation de nos réalisations ou même de respect des droits des auteurs. Depuis une dizaine d'années, la réflexion est engagée. La SER a ainsi pu faire passer la gestion de la revue, des abonnements à la fabrication et à la diffusion, chez un éditeur privé, ce qui a permis de recentrer le travail bénévole sur le contenu lui-même, tout en dégageant des ressources plus régulières. Parallèlement, s'agissant de nos propres éditions, la Société a pu reconsidérer la gestion des stocks existant et surtout les possibilités techniques de réimpression ou de mise à disposition informatique de nos titres épuisés, et en dégager également des ressources supplémentaires, sitôt elles aussi réinvesties. Elle a également initié une politique d'écriture collective d'ouvrages destinés à un public 
large, confiés à des éditeurs privés, en créant là aussi de minces ressources ${ }^{2}$. Les dons ou legs de sociétaires ou d'amis sont pourtant jusqu'à présent restés limités, au fond parce que personne ne voyait clairement à quoi d'utile serviraient de tels dons, même fiscalement déductibles à $66,66 \%$, et encore moins des legs.

\section{Les temps forts de la souscription}

L'annonce de la mise en vente des papiers Robespierre-Lebas confronte brutalement la SER aux conséquences du passé et à ses propres retards : faiblesse de ses réserves, manque de fonds propres, manque de ressources prévisibles. Il apparait de suite que notre Société ne pourra peser, au-delà de pieuses protestations contre la mise en vente de ce qui est évidemment un patrimoine national, qu'en initiant une souscription publique à une échelle bien plus large que celle de ses sociétaires, destinée à faire pression sur les autorités ministérielles. Mais il est tout aussi évident qu'en lançant une telle souscription, les prix monteraient de fait au détriment des maigres budgets du Patrimoine. Qui plus est, la SER semblerait justifier les surenchères les plus folles et, peut-être plus grave encore, inviterait les citoyennes et citoyens à contribuer directement à des dépenses qui auraient dû incomber au Trésor public, auquel chacun est déjà censé contribuer « selon ses capacités ». C'est dire si le débat a immédiatement commencé au sein du Bureau de la SER aussi bien que parmi ses administrateurs et membres. La même chose se produisait simultanément à l'Institut d'histoire de la Révolution française, autour de son directeur Pierre Serna, et dans les instances de la douzaine de sociétés amies qui se lançaient elles-aussi dans l'aventure de la souscription. Le courrier des souscripteurs confirmait rapidement que beaucoup d'entre eux se posaient les mêmes problèmes, quitte à accepter de financer en fonction de leurs moyens, sous réserve d'inventaire, et en demandant si les sommes collectées seraient alors restituées.

Ce moment-là a donc été un premier temps fort, en particulier lorsqu'il est apparu que, malgré notre regrettable manque d'expérience, nous étions les seuls à la fois en légitimité et en capacité de gérer une grande quantité de versements, de chèques et parfois de virements, avec

(2) Michel BIARD (dir.), La Révolution française. Une histoire toujours vivante, Paris, Tallandier, 2010 ; Michel BIARD et Philippe BouRdin (dir.), Robespierre. Portraits croisés, Paris, Armand Colin, 2012 ; Philippe BOURDIN et Cyril TRIOLAIRE (dir.), Enseigner la Révolution française et l'Empire au collège et au lycée, Paris, Armand Colin, en cours de préparation. 
retours. Il fallait en effet que les certificats fiscaux correspondants aient une chance raisonnable d'être en possession des souscripteurs au moment de leur future déclaration d'impôt, un an plus tard. Il allait falloir le faire avec les moyens du bord. Cette inquiétude, qui s'ajoutait aux soucis de fond sur la réussite de la collecte et sur la réalité de la préemption, allait se doubler d'une autre encore, au fur et à mesure que les plafonds envisagés pour la collecte étaient régulièrement dépassés : que se passerait-il en cas d'échec global, lorsqu'il faudrait retourner ou rembourser de multiples chèques ou virements aux souscripteurs? Nous nous trouvions donc mis d'un coup en contact avec un public bien plus large que ce que nous avions jamais rêvé et ce au travers d'une procédure financière bien plus ample que tout ce que nous avions ambitionné de gérer jusque là... mais dans une certaine improvisation, largement due au retard avec lequel nous avions « fait nos gammes » en matière de communication et de collecte. Pas mal de sueurs froides devaient suivre et bien des procédures de détail... mais il est vrai qu'entretemps, l'obstacle principal avait sauté.

Le moment de la préemption par les services de l'État est un second temps fort. La salle de vente Sotheby's se situe au cœur du dispositif du pouvoir, juste en face du Palais de l'Élysée. Même si ce n'est pas loin de la boutique où ont longtemps été maintenues les marques de l'ancien domicile de Robespierre, chez les Duplay, un premier choc est ressenti le jour de la vente. La salle est bien pleine et la SER représentée par son secrétaire général Serge Aberdam. Des amis sont là - notamment du monde associatif et de la politique - comme Jack Ralite - ainsi qu'Hervé Lemoine, directeur du Patrimoine auquel les réorganisations successives de la RGPP donnent les responsabilités d'un quasi « vice-ministre ». Ces deux dernières présences font espérer que l'effondrement des résistances n'est pas certain, mais devient possible. Les ventes commencent ; viennent bientôt les deux lots qui nous intéressent et que les enchères anonymes des commissaires font rapidement grimper très au-delà de tout ce qu'il était habituel de considérer comme un prix «normal » pour ce type de documents. Lorsque le directeur du Patrimoine prononce pourtant la formule consacrée qui fait valoir les droits de l'État à se substituer à l'acheteur, donc au prix de la dernière enchère, il se produit une sorte de rugissement, qui se répète à l'envie à sa seconde intervention.

Les sommes à trouver dans le délai théorique de quelques semaines imparti par la loi pour gagner réellement sont astronomiques. Hervé Lemoine fait alors un geste qui marque la colère que lui inspire la façon dont les services de l'État viennent de se «faire tondre ». Il se lève sans autre discrétion et gagne le hall d'entrée mais, dans ce mouvement, il est 
suivi par les deux-tiers de la salle. Votant ainsi avec leurs pieds, les frères, sœurs et amis se découvrent plus nombreux qu'ils n'espéraient et marquent la méfiance, pour ne pas dire plus, que leur inspirent cette vente et ses animateurs. Le sentiment semble partagé et c'est dans une cordiale hostilité réciproque que s'organisent parallèlement, dans la salle, l'interminable suite de la vente et, dans le hall, une sorte de longue conférence de presse à plusieurs voix qui aura une grande importance pour la suite. En effet, c'est là, dans un joyeux désordre, que nous rencontrons pour la première fois non plus seulement des représentants isolés de la presse écrite, mais ceux de l'audiovisuel et singulièrement des radios qui profitent de l'occasion pour enregistrer du matériel ; celui-ci sera diffusé et rediffusé dans les jours suivants, intensifiant l'appel à la souscription que tous les intervenants répètent désormais à l'envi.

Dès lors, il devient nécessaire de passer chaque jour ou presque à notre casier postal en Sorbonne. Aucune liasse n'est énorme mais le casier n'est pas grand et, surtout, il faut suivre les remontées pour leur donner écho et si besoin réponse. Nouveau temps fort. Le soir, le secrétaire général, son épouse et ses deux filles ouvrent le courrier, repèrent les discordances d'adresses et font les comptes. Surtout, c'est le moment où se mesure l'intensité, l'implication profonde qui ressort de beaucoup de courriers. C'est une découverte mais aussi une interpellation. Il y a des lettres dont le ton, ou bien l'âge et la conviction du donateur, semblent appeler une réponse immédiate. Certaines de ces réponses sont rédigées sur le moment même, aux petites heures de la nuit, pour atteindre au plus vite une institutrice depuis longtemps retraitée ou le pensionnaire d'un lointain hôpital rural qui ont répondu à notre appel et envers qui nous éprouvons subitement un vif sentiment de fraternité.

Plus de dix jours se sont écoulés depuis la préemption de l'État chez Sotheby's lorsque Serge Aberdam est invité à venir porter la parole de la SER dans une célèbre émission d'histoire du matin sur France Culture. À sa propre grande surprise, il est confronté à Frédéric Mitterrand, ministre de la Culture et de la Communication, et à la présence, peut-être plus impressionnante encore mais tout aussi surprenante, de Pierre Nora. On débat : le ministère a-t-il fait tout ce qu'il pouvait ? Les papiers auraient-ils pu faire l'objet d'une démarche plus hardie, d'une véritable intervention, au final bien moins coûteuse ? C'est aux auditeurs du service public de se faire leur opinion, et la suite des courriers en témoignera : mais le débat change désormais de niveau, comme la définition des papiers dont il est désormais admis qu'ils doivent devenir patrimoine public. Dans ce nouveau contexte, une remarque malicieuse de Pierre Nora sur 
l'absence de toute rue Robespierre à Paris entraîne une brève discussion qui aura elle-même ses prolongements : peut-être l'historien des lieux de mémoire en serait-il surpris, mais c'est de là que découlera finalement l'idée de commémorer l'anniversaire des 220 ans de la République et de la Convention, le 21 septembre 2012, devant le groupe monumental de Sicard, au centre de la nef du Panthéon; là où figurent entre autres, et plus grands que nature, Robespierre, Danton et Marat.

\section{Kaléidoscope sociologique d'une action militante}

L'estimation des deux lots de manuscrits inédits de Le Bas et de Robespierre avancée par Sotheby's et l'imminence de leur vente impliquent une levée de fonds quasi immédiate. Le 18 mai, lorsque l'État préempte l'ensemble au prix du marteau, à $979400 €$, commission d'achat incluse, ce sont déjà plusieurs dizaines de milliers d'euros qui ont été adressés à la SER. Le compte n'y est évidemment pas et la somme, quoique jugée « très raisonnable » par le commissaire de la vente, Alain Renner, au vu de l'intérêt historique de ces documents rarissimes, atteint près de trois fois l'estimation initiale. Avant le lancement de cette souscription, jamais les « brouillons » de Robespierre n'ont bénéficié de la part d'aucune institution publique d'un programme de levée de fonds, analogue à celui lancé par Le Louvre en faveur des Trois Grâces de Lucas Cranach. Or, si quelque 7200 particuliers et entreprises ont déboursé près du quart du million ${ }^{3}$ nécessaire à l'acquisition du Trésor national de Cranach l'Ancien, ce ne sont pas moins de 1065 particuliers et associations qui réunissent $118490 €$ pour préserver les papiers de l'Incorruptible, soit près de la moitié de la somme initialement évaluée par Sotheby's. Une gageure, un tour de force citoyen, tant l'unanimisme est moins partagé qu'autour de l'œuvre du maître de la Renaissance ; le climat polémique et parfois historiquement intenable rappelant combien la figure de Robespierre, et au-delà même, la Révolution française, n'ont jamais été véritablement reléguées « au rang des mémoires mortes ou des dossiers clos $»^{4}$.

Quelque 1084 dons sont ainsi enregistrés, dix-sept souscripteurs ressortant sans hésiter leur carnet de chèque à deux ou trois reprises, comme pour mieux doper une souscription dont l'issue ne devait qu'être heureuse. Immédiatement sensibles à l'appel lancé en faveur de la sauvegarde de ces

(3) http://www.louvre.fr/acquisition-des-trois-graces-de-lucas-cranach

(4) Michel Vovelle, « À la résurrection des piques ! », Michel BIARD (dir.), La Révolution française. Une histoire toujours vivante, Paris, Tallandier, 2009, p. 18. 
papiers, treize associations adressent leur don à la SER. Motivés par des liens anciens ou plus actifs avec la société fondée par Mathiez - l'association pour la Sauvegarde de la maison de Saint-Just, les Amis de Robespierre pour le Bicentenaire de la Révolution, la Société d'histoire locale de Compiègne et Nantes Histoire -, par le souvenir de la mobilisation de 1989- les Amis de la Liberté, Villejuif-Vive 89! - ou encore par une même appétence historique scolaire - avec l'Association des Professeurs d'Histoire et de Géographie -, ces dons viennent très rapidement alimenter la souscription ${ }^{5}$. L'ambition du rachat est d'ailleurs très clairement annoncée par les trois dons majeurs - de 3 à $10000 €$ chacun - consentis par la SER - en vertu de « son attachement à Robespierre », selon le mot d'Albert Soboul ${ }^{6}$, et de son travail permanent de publication critique de ses écrits ${ }^{7}$-, le Comité de Vigilance sur les Usages Publics de l'Histoire - protestant ainsi vigoureusement contre les usages privés des sources d'une Histoire appartenant à tous ${ }^{8}$ - et la Fondation Gabriel Péri - fidèle à son souci de compréhension de l'Histoire en dehors de toute instrumentalisation ${ }^{9}$. Ces sommes conséquentes seraient pourtant sans efficacité si elles n'étaient complétées par un flot continu de dons, fonction des moyens des souscripteurs et, témoins, parfois, de la conscience d'un intérêt général placé au-dessus de modesties sociales particulières évidentes : cette dame de quatre-vingts ans, cette autre, nouvellement retraitée, « trop pauvre pour payer des impôts », ou bien encore cette jeune étudiante, chacune s'excusant de son " petit don ». Selon un rapport de 1 à 400 , les dons des particuliers ${ }^{10}$ oscillent de $5 €$ à $2000 € .53$ sont compris entre $5 €$ et $15 €, 257$ entre $20 €$ et $35 €, 292$ entre $40 €$ et $55 €, 51$ entre $60 €$ et $85 €, 308$ entre $100 €$ et $150 €, 66$ entre $200 €$ et $250 €, 43$ entre $300 €$ et $500 €$; seuls onze dépassent les $1000 €$. Finalement, $32 \%$ sont inférieurs ou égaux à $40 €, 60 \%$ à $85 €$, et au-delà encore, $88 \%$ à $150 €$; une répartition par tranche largement conforme à la pratique du don caritatif en France où un don sur quatre ne dépasse pas $40 €$ et la moitié n'excède pas $85 €^{11}$. Encouragés par le statut d'utilité publique de

(5) Sans oublier également ceux adressés par la Société d'Études et de Recherches Historiques en Psychiatrie, le Groupe d'étude du matérialisme relationnel et l'association Maximilien Robespierre pour l'idéal démocratique.

(6) Julien LOUVRIER, « Albert Soboul et la Société des études robespierristes », AHRF, juillet-septembre 2008, «Un siècle d'études révolutionnaires 1907-2007 » n³53, p. 229.

(7) Voir les onze tomes des Euvres complètes de Robespierre publiés par la SER.

(8) http://cvuh.blogspot.fr/2011/05/il-faut-sauver-robespierre-appel.html.

(9) Fondation Gabriel Péri. Lettre de M. Robert Hue, Président et Sénateur du Val d'Oise, à M. le ministre de la Culture et de la Communication, Pantin, le 12 mai 2011.

(10) Voir documents $n^{\circ} 1$ et $n^{\circ} 2$.

(11) http://insee.fr. INSEE. « Donner aux organismes caritatifs. Est-ce seulement une question de niveau de vie ? », enquête réalisée par Élise Amar et Solveig Vanovermeir, 2005. 
la SER, les donateurs n'évoquent cependant que rarement l'avantage fiscal accompagnant leur geste; si les mots joints à leurs chèques trahissent, par le papier sur lequel ils sont écrits ${ }^{12}$, une pratique déjà éprouvée du don, certains souscripteurs revendiquent au contraire leur droit à ne pas recevoir leur reçu.

Car cette générosité est avant tout militante, comme en témoignent ces chèques investissant sciemment l'imaginaire collectif révolutionnaire. Ainsi, tandis que ces dons de $179,20 €$ et $93 €$ honorent clairement la fondation de la République et la politique patriotique de l'an II, ces deux autres de $36 €$ ou celui-ci de $68 €$ rendent hommage à ces moments où le pouvoir populaire se cherche. Par-delà les symboles chiffrés, le geste est aussi l'occasion d'affirmer, par l'envoi de son chèque, une culture populaire révolutionnaire contemporaine. Apparaissent, parmi les lots de courriers reçus, ces enveloppes frappées du sceau du bicentenaire, que seule l'exceptionnalité de la situation justifie d'utiliser à nouveau ; ou ces cartes estampillées, pêle-mêle, comme tout droit sortis de la cave ou du grenier révolutionnaire familial, du visage de Montesquieu et de l'Incorruptible, du bonnet phrygien et des faisceaux républicains, ou bien de cette affiche Série 1789 d'Alain Snyers associant rubans et drapeaux tricolores, patriotes et déclaration des droits de l'homme et du citoyen.

Aussi signifiant soit-il, ce soin, somme toute finalement ponctuel, n'efface en rien l'urgence générale du don, imposée par l' «immédiateté » de la vente aux enchères; une urgence et une forme de mobilisation relativement inédite dont rend compte une majorité de dons. Aucun bulletin type n'ayant été diffusé avec l'appel à souscription, chacun se sent d'autant plus libre de la forme de son envoi. L'urgence de la vente pousse près de $70 \%$ des souscripteurs à simplement glisser leur chèque dans une enveloppe, sans aucun commentaire ; l'important étant alors que leur don « ne parvienne pas trop tard » à la SER, comme l'écrit volontiers une donatrice. Les 329 mots accompagnant les envois témoignent eux aussi de ce caractère d'urgence. 109 sont notés sur un simple post-it, 94 autres sur une simple carte de visite ; un mot sur trois seulement - soit 101 sont consignés sur une feuille classique ou une page de papier à lettres ${ }^{13}$. Tandis qu'une trentaine de donateurs se contentent d'indiquer leur adresse, 209 y vont de leurs encouragements, francs mais généralement brefs ; ils

(12) Au moins onze souscripteurs font le choix d'adresser leur chèque avec quelques lignes écrites sur un papier à lettres estampillé Handicap international, Fondation de France, Fondation recherche médicale ou $U N I C E F$.

(13) Ajoutons à ce décompte 25 autres « mots » inscrits sur des cartes diverses. 
sont toutefois 89 à prendre le temps de justifier leur geste, 77 sur une quinzaine de lignes et une douzaine au-delà, de manière beaucoup plus nourrie encore. Autant de «mots » dévoilant alors les motivations, les parcours, les identités de celles et ceux qui se sont immédiatement sentis concernés par la sauvegarde de ces manuscrits.

« Dévoilant» seulement. Car l'immense majorité des donateurs reste cantonnée aux adresses, noms et prénoms figurant sur le chèque envoyé. Le ton humble ou militant, le trait ferme ou l'écriture tremblante, l'en-tête de la carte de visite professionnelle ou le papier à lettres choisi sont pourtant autant d'indices pour dresser un portrait collectif, aussi fin que possible, de ce millier de donateurs. Une "poignée » livre son âge, comme pour mieux inscrire son geste dans le temps d'une vie engagée - une seule le fait pour nourrir « [les] statistiques », se projetant déjà dans l'analyse quasi immédiate d'un mouvement sans précédent. Quel âge a-t-on lorsque l'on dit avoir découvert la Révolution en lisant Mathiez, enfant ? 68 et 70 ans, comme ce couple seine-et-marnais ? Ou davantage, comme cette Parisienne de 83 ans se revendiquant, haut et fort, « toujours révolutionnaire ». André, Madeleine, Fernand, Martine, Renée, Jacques, Monique, Guy, Thérèse. Qui a réceptionné toutes les enveloppes sait combien les prénoms des donateurs journellement égrenés paraissent esquisser un tableau de groupe. Celui d'une ou plusieurs générations qui mesureraient mieux que les plus jeunes le poids de l'histoire et l'impérieuse nécessité de préserver les précieux brouillons de l'Incorruptible. Il serait tout aussi imprudent d'en faire une règle que de refuser aux autres cette conscience historique finalement aiguë. Derrière l'intemporalité supposée des prénoms pointent pourtant de fortes modes susceptibles d'éclairer - avec toute la prudence méthodologique ${ }^{14}$

(14) Le choix d'analyse des prénoms des souscripteurs s'est avéré comme le seul moyen d'esquisser un tableau générationnel des donateurs. Pas inutile en soit, ce portrait de groupe n'en est pas moins « faux », tant, malgré les modes d'attribution, un prénom a généralement une durée de vie plus que variable, s'étendant d'une ou deux décennies au siècle, et davantage encore. L'année attribuée à chaque prénom de souscripteur recensé correspond à celle de son pic d'attribution, entre 1920 année de naissance du plus âgé donateur repéré - et 1992 - cap de la majorité révolue du souscripteur supposé le plus jeune, et en âge de responsabilité bancaire. Voisinant souvent avec l'année médiane d'attribution du prénom au cours d'une période moyenne de 15 à 20 ans marquant une véritable mode, le pic d'attribution a été privilégié. 323 prénoms ont été enregistrés. Une projection mathématique proposant un modèle de répartition de chaque prénom sur le siècle dernier, fonction des périodes de flux et de reflux de chacun, eût évidemment été préférable ; il aurait néanmoins dû être corrigé par les indispensables paramètres socioprofessionnels ou culturels, dont nous ne disposions pas. Nous avons donc compté sur un rééquilibrage partiel des prénoms finalement « non ventilés », entendu que le pic de chacun d'entre eux a été réuni à une bande chronologique de dix ans (2011-1992, 1991-1982, 1981-1972, etc.). Voir Jacques DUPÂQUIER, Jean-Pierre PÉLISSIER et Danièle RÉBAUDO, Le Temps des Jules. Les prénoms en France au XIX ${ }^{e}$ siècle, Paris, Éditions Christian, 1987. 
et la réserve analytique nécessaires - ce « portrait générationnel ${ }^{15}$. Ainsi, quatre souscripteurs seraient susceptibles d'avoir moins de 20 ans, 45 de 20 à 29 ans, 57 de 30 à 39 ans, 201 de 40 à 49 ans, 121 de 50 à 59 ans, 359 de 60 à 69 ans, 37 de 70 à 79 ans et enfin 166 auraient plus de 80 ans. Un peu moins d'un donateur sur trois seulement aurait moins de 49 ans et donc $70 \%$ plus de 50 ans ; une projection finalement « réaliste » tant la part des 50-65 ans - $47 \%$ des souscripteurs - voisine avec celle des donateurs des œuvres caritatives - un peu plus de $50 \%$-, comme celle des plus de 55 ans $-63 \%$ pour les souscripteurs contre $60 \%$ habituellement. La mise en perspective des profils de ces donateurs, largement fonction de l'originalité de la souscription, peut se poursuivre sans doute plus justement avec la pyramide des âges des sociétaires de la SER. Car ils sont en effet 102, membres de la Société des études robespierristes, - sur 249 - à avoir mis la main au portefeuille. Avec $44 \%$ de sociétaires âgés de plus de 60 ans - dont plus de un sur deux a même plus de 70 ans -, $31 \%$ de 50 à 59 ans, $17 \%$ de 40 à 49 ans et seulement $8 \%$ de moins de 39 ans en $2005^{16}$, la structure d'âges de la SER, toujours valable en 2011 malgré l'inévitable mais légère mobilité des lignes et des classes, valide justement celle, projetée, des souscripteurs. Sans jamais retirer pour autant aux plus jeunes, moins représentés, leur capacité à s'investir et se mobiliser ; en témoignent ces étudiants et nouveaux enseignants dénombrés parmi les donateurs.

Seuls 52 indiquent leur situation/profession; et parmi eux, pas moins de 34 enseignants, du secondaire ou de l'université, non membres de la SER, actifs ou retraités. Rien ne dit au final, du reste, que les professeurs forment le plus gros des souscripteurs, et ce, même si parmi la centaine de ces sociétaires-donateurs, ils sont, avec les chercheurs, omniprésents ${ }^{17}$. Sans doute sont-ils même minoritaires tant l'écho de cette souscription dépasse alors les seuls cercles universitaires et scolaires et touchent celles et ceux qui partagent cet intérêt pour la chose publique. Aussi aux côtés d'une ex-principale et d'un ancien élève des collèges Robespierre d'Arras et de Saint-Étienne-du-Ry apparaissent trois hauts fonctionnaires, autant de responsables, en vue, de l'édition et des médias, de conservateurs et de directeurs de services publics d'archives, d'avocats

(15) Voir document $n^{\circ} 3$.

(16) Enquête menée sur un échantillon de 133 sociétaires en 2005 en perspective de la célébration du centenaire (en 2007) de la SER. Michel BIARD, «La composition actuelle de la Société des études robespierristes », AHRF, op. cit., n³53, p. 19-27.

(17) Ibid., p.21. 
et de médecins, un historien bankable, un libraire, un graphiste, un chômeur et quatre élus nationaux - qui ont joint le geste à la parole, prise publiquement dans les hémicycles ou relayée dans leur circonscription ${ }^{18}$. Derrière l' «impossible » tableau socioprofessionnel de ce millier de donateurs apparaissent néanmoins, en filigrane, les réseaux associatifs, militants, d'amitiés qui se sont alors activés.

Signe d'une sensibilité commune, au moins 39 souscripteurs partagent des liens familiaux ${ }^{19}$; père/fils (le), frère/sœur, mari/épouse, le mot s'est donc parfois donné en famille, chacun y allant, jusque dans le même ménage parfois, de son propre don. En association, le don collectif a été, de temps à autre, redoublé de gestes personnels, comme aiment à le rappeler ces divers adhérents de l'ARBR, de Nantes Histoire, des amis de la Maison de Saint-Just ou, évidemment, de la SER ; pour amplifier le mouvement et mieux signifier encore la somme des volontés mobilisées. Vu les liens étroits tissés et entretenus entre les milieux de la gauche, principalement communiste, et certains des membres de la SER au fil des décennies ${ }^{20}$ sans que, par ailleurs, ne puisse être remise en cause sa véritable ouverture politique, comme à ses origines ${ }^{21}$, il n'est finalement pas surprenant de voir combien certains réseaux se sont efficacement activés à cette occasion. Vingt-deux souscripteurs sur trente-quatre signalent ainsi avoir pris connaissance de cette vente dans les colonnes de l'Humanité, à travers les tribunes successives de Pierre Serna puis Philippe Bourdin. Plusieurs donateurs revendiquent leur appartenance au Parti de Gauche et au Parti Communiste Français - dont l'un d'eux, à ce dernier, depuis 1947 - ; certains militants joignent à leur courrier le montant des sommes rassemblées lors d'une réunion de section - du Sud parisien notamment -, relayant de la meilleure façon les prises de position quasi immédiates des états-majors ou secrétariats nationaux communiste et socialiste. Anciens adhérents (re)connus et simples militants actifs des partis de la gauche (alors) d'opposition 《extrême ${ }^{22}$ ou plus modérée - adressent leur don avec enthousiasme et diffusent l'information. Nombreux sont aussi les sympathisants de cette «gauche sociale » à saluer, pour l'un, « une initiative de salut public »

(18) En particulier Mme Catherine Lemorton, députée PS de Haute-Garonne, et M. Jack Ralite, alors sénateur PCF de la Seine-Saint-Denis (rassemblement CRC, « Communiste, Républicain, Citoyen » au Sénat).

(19) Au moins 19 « paires » ou « trio » connus ou présentés explicitement ont été formellement identifiés comme étant des mêmes familles.

(20) Voir le numéro spécial des AHRF, op. cit., n³53.

(21) Philippe BOURDIN, «Introduction » op. cit., n³53, p. 9.

(22) Michel Biard, Bernard GAINot, Paul PASTEUR et Pierre SERnA (dir.), « Extrême »? Identités partisanes et stigmatisation des gauches en Europe (XVIII $-X X^{e}$ siècle), Rennes, PUR, 2012. 
[sic], ou à accompagner leur don, pour les autres, de leur « amical salut public », de leurs " amitiés républicaine et laïque », de leurs « sentiments fraternels », ou bien du plus simple et républicain « salut et fraternité ». Car derrière les mots des uns et des autres se dessine un discours citoyen engagé, naturellement pluriel, reflet tout à la fois d'une préoccupation patrimoniale au-dessus des partis, d'un respect, souvent admiratif, de l'Incorruptible, et d'une réelle indignation, culturelle, sociale et politique.

\section{L'éventail des motivations, entre indignation et devoir d'Histoire}

La singularité de cette souscription appelle inévitablement des motivations personnelles très variées et que les «mots » joints aux dons dévoilent, dès lors qu'ils dépassent les plus sommaires encouragements. Plus d'un sur deux exprime le vif désir de voir les manuscrits sauvés entrer dans les collections publiques. En affirmant qu'il s'agit d'un vrai « patrimoine national » dont la France ne doit en aucune manière être privée, les donateurs reprennent aussi les vœux formulés dans les tribunes publiques de Pierre Serna et Philippe Bourdin, l'appel à souscription diffusé par la SER, les communiqués du PCF et du PS ou encore les mots du directeur du Patrimoine Hervé Lemoine évoquant un «trésor patrimonial ${ }^{23}$. Le phénomène reflète l'ascendance du processus de patrimonialisation des politiques culturelles actuelle ${ }^{24}$ et sa large assimilation par l'opinion. Pour beaucoup, le plus incompréhensible demeure l'inertie de l'État, son immobilisme initial ou sa «[regrettable] carence ou défaillance $»^{25}$; sa mobilisation à retardement pour la préservation des papiers de l'un des acteurs majeurs de la Révolution française. Nombre d'intervenants condamnent sa mise à l'index - déjà clairement ressentie dans la synthèse historique globalisante du Président d'alors, vantant une République mue par une continuité de principes, décontextualisée et niveleuse, la République des Lamartine, Gambetta, Clémenceau et de Gaulle, qui ne serait pas celle de Danton et Robespierre ${ }^{26}$. L'émoi et la mobilisation, la pression d'historiens,

(23) Audrey PELÉ, « La France va garder les manuscrits de Robespierre », Le Figaro, édition du 18 mai 2012.

(24) François Hartog, Régimes d'historicité. Présentisme et expérience du temps, Paris, Seuil, 2003, p. 137 et $s q$.

(25) Archives SER, lettre du Groupe d'Étude du Matérialisme Rationnel à la Société des études robespierristes, le 15 mai 2011.

(26) Marc BELISSA, «Révolution française et "ses grands hommes", Laurence DE COCK, Fanny MADEline, Nicolas OfFEnSTADT et Sophie WAHnich (dir.), Comment Nicolas Sarkozy écrit l'histoire de France, Marseille, Agone, 2008, p. 171-174. 
d'associations et d'élus vigilants ainsi que la prise de conscience des plus hautes instances culturelles évitent alors la perte des manuscrits.

Le faisceau des motivations des souscripteurs reflète ainsi autant qu'il nourrit un discours d' « indignation » - un argument sur sept recensé, soit 34 des 230 recensés. Le motif du don est alors l'indignation, en parfait écho au cri lancé à l'octobre 2010 par Stéphane Hessel. Si le retentissant petit livre de l'ancien Résistant est bien « cet objet dont se saisit le présent », selon le mot de Nicolas Offenstadt ${ }^{27}$, alors nombre de citoyens s'indignent bien du sort réservé aux manuscrits de Robespierre. «Indignée » comme cette chômeuse ne pouvant donner plus de $10 €$, cette militante encartée mue par des «principes de progrès » ou cette autre révulsée par cette spéculation patrimoniale :

«Que l'on apprécie ou que l'on déteste tel aspect, tel moment, tel personnage de la Révolution française, ne saurait avoir d'influence sur le sort et l'étude de tous documents historiques. Que des documents de [cette] importance $[. .$.$] se retrouvent dans des mains privées alors qu'ils devraient$ depuis fort longtemps avoir été confiés à la Bibliothèque Nationale, cela me bouleverse. Et que le pire outrage qui leur soit fait devienne un enjeu de propriété privée, c'est indigne ! Donc je m'indigne ! $»^{28}$.

Cette indignation, plurielle, se cristallise alors autour de la propriété résolument nationale des manuscrits ${ }^{29}$; au nom de la place incontestable que Robespierre occupe au Panthéon républicain historique français, ces papiers appartiennent et reviennent moralement au peuple selon certains ${ }^{30}$. Assimilant tout à la fois la république, le peuple, la démocratie et/ou l'État, les donateurs élèvent la patrimonialité et l'inaliénabilité des documents privés d'une figure publique majeure de l'Histoire nationale au rang de principes inviolables ${ }^{31}$. L'argumentaire est développé par d'autres qui condamnent «leur soumission aux lois du marché $»^{32}$, selon une

(27) Stéphane HEssEL, Indignez-vous !, Paris, Indigène éditions, 2011 (édition revue et augmentée).

(28) Archives SER, lettre d'une donatrice, le 13 mai 2011.

(29) Ibidem, lettre d'une donatrice, [non datée]. « Bien que j'ai beaucoup de mal à comprendre pourquoi les Archives Nationales doivent acheter un bien qui est « national », voici notre contribution (modeste). [...]».

(30) Ibid., lettre d'un donateur, le 14 mai 2011. « [...] Je joins une contribution de $300 €$ qui, j'espère, contribuera à conserver les manuscrits qui appartiennent moralement à notre démocratie et à son peuple ».

(31) Archives SER, lettre d'une donatrice, le 9 mai 2011. « [...] Il faut qu'il y ait un relais politique pour une éventuelle préemption, au moins en soutien de la part du gouvernement, une prise de position sur ce patrimoine qui devrait être inaliénable puisque appartenant à la Nation ».

(32) Ibid., lettre d'une donatrice, [non datée]. 
rhétorique (re)connue commune aux forces de la gauche, révolutionnaire et/ou républicaine, extrême ou contestataire - dont l'intérêt va alors grandissant dans une partie de l'opinion, à un an d'un nouveau scrutin présidentiel, et dont le succès, palpable, galvanise ${ }^{33}$ les futurs candidats déjà déclarés et influe bientôt sur leurs prises de paroles publiques ${ }^{34}$. Car cet appel à souscription, réaction à l'apathie publique initiale, apparaît alors à quelques-uns telle une défiance ; comme si, cette république qui a favorisé les nantis - bouclier fiscal, abattements et exonérations en tout genre, dépénalisation du droit des affaires, régulation promise mais enterrée des bonus et autres stock-options ${ }^{35}$ - se montrait incapable d'user des deniers publics pour préserver le bien commun. Les spéculations promises par les enchères sur des documents patrimoniaux concentrent ainsi les rancœurs contre « les fripons $»^{36}$, « les cupides $»^{37}$, les « riches [accapareurs] $»^{38}$, les « possédants $»^{39}$, « les forces de l'argent $»^{40}$ ou $«$ les tenants du capital $»^{41}$. Cette dénonciation rejoint la crainte formulée par le directeur de l'Institut d'histoire de la Révolution française, dans Le Monde, de voir les lots Lebas et Robespierre «terminer dans des fonds privés à l'étranger, ou plus humiliant, [être] acquis par une bibliothèque hors de France $»^{42}$, peur explicitement partagée par une douzaine de donateurs. Pour eux, et dans l'esprit de la prise de position du Comité de Vigilance des Usages de l'Histoire, «le savoir n'est pas une marchandise ». Pas davantage que la culture ou bien l'école de plus en plus contraintes à « la norme sociale du capitalisme $»^{43}$, comme l'écrit cette Arlésienne ${ }^{44}$ ou le crie cette Toulonnaise :

« [...] Notre Nation, ses services publics, son domaine public partent en lambeaux, vendus par les uns, achetés par les autres, au nom du fric roi ! Cette « royauté » n'a pas été abolie... J'hésite à peine à affirmer que de la spéculation à la corruption, il n'y a guère que quelques pas de côté à

(33) Raphaëlle Besse Desmoulières, «Jean-Luc Mélenchon avec cocarde et bonnet phrygien », Le Monde, le 19 mai 2012.

(34) Voir Discours du Bourget de François Hollande, le 22 janvier 2012.

(35) Michel PInÇOn et Monique PInÇOn-CHARlot, Le Président des riches. Enquête sur l'oligarchie dans la France de Nicolas Sarkozy, Paris, Zones, 2010.

(36) Archives SER, lettre d'un donateur, [non datée].

(37) Ibid., lettre d'une donatrice, [non datée].

(38) Ibid., lettre d'une donatrice, le 16 mai 2011.

(39) Ibid., lettre d'une donatrice, le 17 mai 2011.

(40) Ibid., lettre d'une donatrice, le 13 mai 2011.

(41) Ibid., lettre d'un donateur, le 17 mai 2011.

(42) Pierre SERNA, « Il faut sauver le soldat Robespierre ! », art. cit.

(43) Christian Laval, Francis Vergne, Pierre Clément et Guy Dreux, La nouvelle école capitaliste, Paris, La Découverte, 2011.

(44) Archives SER, lettre d'une donatrice [1], le 13 mai 2011. 
faire. Qu'écrirait aujourd'hui l'Incorruptible ? Qu'avait-il pu relater qu'il dénoncerait prophétiquement les pratiques actuelles de ventes aux enchères, donc au plus offrant ? [...] $\gg^{45}$.

Aussi le don de quelques-uns est-il bien à mettre au rang d'une résistance citoyenne, d'une forme d'insurrection civique contre le règne supposé/avéré de l'argent - bientôt érigée en modèle républicain, de la Bastille à la plage du Prado. L'incorruptibilité de Robespierre force à l'évidence l'admiration de certains, alors même que la SER - conformément au souhait de Mathiez ${ }^{46}$-n'a jamais engagé cette souscription publique pour entretenir la flamme en l'honneur d'aucune idole, et que ses membres ne se revendiquent pas tous robespierristes - certains se préférant « jacquesrouxtins », d'autres cultivant la figure de Saint-Just, voire aucune, très simplement. Ils sont au moins vingt-et-un souscripteurs à écrire tout le bien qu'ils pensent de Robespierre et motiver ainsi leur geste. « Droit », « vertueux », « exceptionnel », « le plus grand révolutionnaire français » est loué comme le « serviteur du peuple » ${ }^{47}$, apprécié comme celui qui « voulut abolir l'esclavage, accorder le droit de vote à tous y compris aux femmes, limiter l'enrichissement des bourgeois et imposer la loi sur le maximum ${ }^{48}$; il est de fait «l'idole de l'adolescence » ou « l'amour « constituant » » de ces deux donatrices, telle autre retraitée de la Poste avouant qu'elle « a un grand faible » pour lui ${ }^{49}$. Faudrait-il donc aimer Robespierre pour vouloir sauvegarder les brouillons de ses discours? Son souvenir ne saurait évidemment être accaparé, pas davantage que l'accès à ses papiers réservé à quelques-uns. Plusieurs, vingt-six exactement - soit près d'un donateur sur trois qui se justifie plus longuement -, souhaitent ainsi entretenir la mémoire de l'Incorruptible ou promeuvent le devoir d'Histoire. Si celles et ceux qui, par leur geste, effectué en souvenir des historiens majeurs qui leur ont fait (re)découvrir et comprendre la Révolution française Albert Soboul (7), Michel Vovelle (3) ou Claude Mazauric (3) $)^{50}-$, dans les livres ou sur les bancs des universités, ou, qui en appellent à une nouvelle historiographie de « combat », répondant aux assauts menés, hier, contre une lecture « classique » de la période par François Furet et Mona

(45) Ibid., lettre d'une donatrice [2], le 13 mai 2011.

(46) Philippe BOURDIN, «Introduction », AHRF, op. cit., n' 253 p. 7.

(47) Archives de la SER, lettres de donateurs, du 14 au 28 mai 2011.

(48) Ibid., lettre d'une donatrice, le 17 mai 2011.

(49) Ibid., lettres de donatrices, [non datées].

(50) Notons que d'autres anciens élèves « repérés » parmi les donateurs n'invoquent pas ces figures au moment de glisser un mot, ou simplement leur chèque, dans l'enveloppe. 
Ozouf ${ }^{51}$, pourraient être rangés derrière les robespierristes, nombre ne le sont pas ; mais participent volontiers à la souscription, afin que l'Histoire s'écrive objectivement et de façon dépassionnée, afin qu'il s'en dégage une certaine vérité qui ne puisse être manipulée, détournée ou simplifiée ${ }^{52}$. « Il n'est pas nécessaire d'être robespierriste pour faire avancer l'histoire de la Révolution française, et même pour beaucoup est-il préférable de ne pas l'être » expliquait, il y a peu, Vovelle ${ }^{53}$. Ainsi en va-t-il de cette figure reconnue de l' « école critique » de l'histoire de la Révolution, de cet autre rappelant combien « Dieu sait qu' [il n'est] pas robespierriste $»^{54}$ ou bien de celui-ci, soucieux de voir « [conservés] en France et [mis] à la disposition des historiens les écrits de Robespierre menacés », précisant que « son geste ne doit pas être interprété comme un geste de sympathie à l'égard du personnage, au contraire $»^{55}$. Certains espèrent les manuscrits pour le musée d'histoire de la Révolution de Vizille, d'autres pour le futur musée d'Histoire de France, la plupart pour les Archives Nationales ou la Bibliothèque Nationale de France. Quelques-uns y voient l'occasion de réactiver la mémoire de Robespierre dans l'espace public, regrettant vertement ici, à Fontenay-le-Fleury, que la rue au nom de l'Incorruptible ait été débaptisée au profit de Pompidou, à l'occasion d'un changement de majorités ${ }^{56}$, ou soutenant ailleurs, à Paris notamment, l'attribution d'une place à Robespierre ${ }^{57}$.

Cette quête de l'Histoire ou cette perpétuation de la mémoire de l'Incorruptible témoignent également de rapports personnels à l'objet les manuscrits -, à la figure historique - en général, et à Robespierre en particulier, ici - et à l'Histoire. Plusieurs (5) souscrivent aux noms de leurs enfants ou de leurs petits enfants, suggérant en retour un « certificat» authentifiant leur geste, et ce, pour que Thibault, Anaïs et Aël « aient chacun un "morceau" de [1'] histoire de France $»^{58}$ ou encore que Clotilde et Axel "puissent comprendre [le] passé $»^{59}$. Souscrire revient pour certains à valider un parcours de vie qui croisa ou se devait finalement de croiser celui

(51) Ibid., lettre d'une donatrice, le 28 mai 2011.

(52) Sophie WAHNICH, « Révolution française, révolution », dans Laurence DE COCK, Fanny MAdeline, Nicolas OfFENSTADT et Sophie WAHNICH (dir.), Comment Nicolas Sarkozy écrit l'histoire de France, op. cit., p. 167-170.

(53) Michel VovelLE, « À la résurrection des piques ! », Michel BIARD (dir.), La Révolution..., op. cit., p. 25

(54) Archives SER, lettre d'un donateur, le 27 mai 2011.

(55) Ibid, lettre d'un donateur, le 15 mai 2011.

(56) Ibid., lettre d'un donateur, le 30 mai 2011.

(57) Ibid., lettre d'un donateur, [non datée].

(58) Ibid., lettre d'un couple de donateurs, le 19 mai 2011.

(59) Ibid., lettre d'un couple de donateurs [1], le 26 mai 2011. 
de Robespierre. Cette souscription s'impose alors comme l'occasion unique d'une rencontre personnelle ou familiale ; à l'image de ce Palois, heureux de voir sa «famille de petits paysans béarnais » rejoindre le cours de la « Grande Histoire ${ }^{60}$. Une dizaine agit en mémoire d'un instituteur, d'un professeur de collège, d'un père, d'une mère ${ }^{61}$, d'un mari qui affectionnait l'Incorruptible, et à qui l'occasion est offerte de rendre hommage, tout en affichant le désir de manifester un attachement individuel renouvelé au grand homme. Au révolutionnaire pour certains ; en vertu d'une histoire familiale engagée, pour ce couple dont les aïeux furent guillotinés en thermidor an II, déporté en nivôse an IX ou tombé en 1819 sous les ordres de Bolivar pour la liberté de l'Amérique du Sud ${ }^{62}$. Au républicain, pour cet autre, fier de sa trajectoire et soucieux d'une forme certaine de réhabilitation de Robespierre.

« Je suis né en 1973 de parents immigrés portugais et mon passage par l'école républicaine m'a permis d'apprécier les valeurs de la République et le rôle de Robespierre pour les installer et les défendre. Je tiens à vous remercier pour ce que vous faites en espérant qu'un jour notre héros commun ne soit plus réduit au cruel dictateur sanguinaire comme il est couramment décrit ${ }^{63}$.

Histoires, parcours, profils et motivations varient mais d'aucun ne s'écarte de la sauvegarde de ces manuscrits inédits dans les collections publiques ; chaque don nourrissant une mobilisation d'ampleur nationale, observée sur tous les points du territoire de la République.

\section{Un mouvement d'ampleur nationale}

La couverture médiatique quasi immédiate permet à l'appel à souscription de rencontrer un important écho dans tout le pays. Les chaînes et radios d'information nationales (reportages, interviews de Pierre Serna et Philippe Bourdin, sur TF1 et France 2 dès les 12 et 13 mai ; focus de France 3 Régions-Arras le lendemain; enquête des équipes d'Europe 1 le 24 mai ; passage de Serge Aberdam sur France Culture le 27 mai,

(60) Ibid., lettre d'un couple de donateurs, le 27 mai 2011.

(61) Ibid., lettre d'une donatrice, le 26 mai 2011. « Ci-jointe la participation de ma mère [...] à la souscription pour les manuscrits de ROBESPIERRE. Ma mère est décédée l'été dernier mais je sais combien il aurait été important pour elle de contribuer - même modestement - à cet élan national envers un des grands hommes de notre histoire. Je vous remercie de faire apparaître son nom sur la liste des donateurs, le mien y étant déjà ».

(62) Ibid., lettre d'un couple de donateurs [2], le 26 mai 2011.

(63) Ibid., lettre d'un donateur, le 27 mai 2011. 
dans l'émission d'Emmanuel Laurentin, La Fabrique de l'Histoire), la presse nationale (L'Humanité, France Soir, Le Monde, Libération, Le Figaro) et les quotidiens régionaux (Le Parisien et Nice Matin le 18 mai) amplifient le mouvement en relayant l'information. Celle-ci est encore très largement diffusée sur la toile, par mails, auprès des sociétaires de la SER qui la relaient à leur tour à la « vitesse du fluide électronique »; vecteurs privilégiés d'une information désormais instantanée, les réseaux sociaux sont au même moment investis pour la première fois par la SER qui ouvre coup sur coup, avec Julien Louvrier, des comptes Facebook et Twitter (34 posts effectués sur la page du premier entre les 11 mai et 3 juin et auxquels ont accès immédiatement et directement plus de 150 personnes, davantage encore par capillarité informatique).

Si le mouvement des dons est bien réellement d'ampleur nationale ${ }^{64}$, ceux-ci se répartissent très inégalement sur le territoire. Si Paris (243) et la région parisienne (227) représentent presque la moitié des donateurs (44\%) et plus de la moitié des dons cumulés $(58,5 \%$, soit un peu plus de $65974 €$ ), les souscripteurs parisiens donnent davantage, entendu que le montant total de leurs dons est presque le double de celui des autres Franciliens (43903€ pour les premiers contre quelque $22071 €$ pour les seconds) ${ }^{65}$. Cette surreprésentation du don des habitants de la capitale, et au-delà de l'île-de-France, n'est pas surprenant tant il est particulier à la pratique du don, de manière générale, dans le pays ${ }^{66}$, et tant les premiers réseaux militants activés furent parisiens. Les autres régions comptent moins de cinquante donateurs chacune à l'exception des régions RhôneAlpes (67) et Provence Alpes Côte d'Azur (87), où la tribune publiée dans Nice Matin, et à laquelle plusieurs souscripteurs font référence, a eu un réel effet mobilisateur. Aucune région n'échappe au mouvement et ce, même si, en Corse (1) comme aux Antilles (3), les donateurs ne se comptent que sur les doigts d'une seule main. Douze régions comptent entre 9 et 20 souscripteurs, quatre entre 21 et 30 , quatre également entre 31 et 40 ; le Midi-Pyrénées, le Nord-Pas-de-Calais, le Centre et l'Aquitaine arrivant ainsi derrière le quatuor de tête des régions les «plus généreuses ».

(64) Seize dons sont enregistrés depuis l'étranger et apparaissent le plus souvent comme autant de gestes de soutien d'enseignants-chercheurs spécialistes de la Révolution française, adhérents ou proches de la Société des études robespierristes ; ils se répartissent ainsi : États-Unis (4), Royaume-Uni (2), Japon (2), Canada (1), Liban (1), Suisse (1), Espagne (1), Finlande (1), Australie (1), République Tchèque (1), Monaco (1).

(65) Que Jean-Paul Rothiot soit ici chaleureusement remercié pour les nombreuses statistiques régionales qu'il a bien voulu nous proposer et pour les projections graphiques et cartographiques qu'il a réalisées, à partir du fichier postal des souscripteurs.

(66) http://insee.fr. INSEE. « Donner aux organismes caritatifs... », op. cit. 
Le poids démographique de chaque région ne semble pas être un facteur déterminant pour expliquer la répartition des dons. Le Limousin compte ainsi autant de donateurs que la Lorraine alors même que sa population est trois fois moins nombreuse. La ville de Paris offre un ratio de 114 donateurs pour un million d'habitants, là où la région parisienne, quatre fois plus peuplée, en a, elle, 27 pour un million. Ce facteur population n'est toutefois pas à exclure puisque, à ratio égal, le Limousin a 15 donateurs et la région Provence-Alpes-Côte-d'Azur 87. Le décompte par département met en évidence le contraste entre certains où plusieurs dizaines d'individus se sont manifestés ( 22 en Haute-Garonne, 23 en Loire-Atlantique, 32 dans le Rhône ou encore dans les Bouches-du-Rhône) et ceux où l'on a très isolément répondu à l'appel (un seul dans le Lot-et-Garonne et en Corrèze, deux en Indre-et-Loire et en Haute-Marne). Les premiers correspondent aux plus grandes métropoles régionales (même si Nancy, Strasbourg ou Caen sont nettement en retrait), aux grandes villes où des centres de recherches universitaires sur la Révolution française sont actifs (Lille, Rouen, Clermont-Ferrand, Lyon, Toulouse et Aix-Marseille) ; ce sont aussi des départements où vivent des associations qui cultivent la mémoire de la Révolution française et concourent à renouveler et faire connaître son histoire. La répartition de ce millier de donateurs sur autant de territoires locaux et sa forte concentration dans certains pôles urbains (un sur deux à Lyon pour le Rhône ou à Marseille pour les Bouches-du-Rhône) ne doit pas empêcher de pousser plus loin encore l'analyse de ces cartes des donateurs. Les disparités régionales pointées révèlent finalement une géographie originale du mouvement, validant ici ou infirmant là, celle, largement admise, des mentalités et des cultures sociopolitiques françaises, nationales et/ou locales.

Le faisceau des motivations exprimées par les souscripteurs renvoie essentiellement - mais non pas exclusivement - à une France indignée, militante, partageant une sensibilité politique et sociale de gauche - dans son acception la plus plurielle. Aussi, la représentation cartographique des dons valide-t-elle une projection finalement très politique ? Le décompte départemental doit être évidemment manié avec prudence (tant les écarts entre les départements où l'on donne le plus et le moins se chiffrent à quelques dizaines d'unités près) ; sans pour autant interdire la possibilité de le confronter avec les nouvelles données électorales enregistrées depuis le rachat, et notamment au printemps 2012. L'espace régional le plus rétif au don pour sauvegarder les manuscrits de l'Incorruptible renvoie incontestablement aux franges bleues traditionnellement à droite du Massif Central et historiquement réticentes à l'acculturation révolutionnaire (le 
Cantal, la Haute-Loire et la Lozère sont les seuls départements contigus où aucun don n'est recensé). Les dons en nombre très limité enregistrés en Basse-Normandie (9), en Champagne-Ardenne (10), en Alsace (10) ou encore en Lorraine (16) paraissent confirmer que l'on se mobilise moins autour de cette sauvegarde dans les départements à forte tradition politique conservatrice, renvoyant alors Robespierre à une figure dont seules les «terres de gauche» devraient pouvoir cultiver l'histoire. Paraissent seulement, car certains des départements du Sud-ouest, fortement ancrés à gauche, n'ont pas donné davantage que les précédents (cinq donateurs pour les trois départements du Gers, du Tarn-et-Garonne et du Tarn), alors que la région Provence-Alpes-Côte-d'Azur, bien plus à droite, enregistre un nombre de dons largement supérieur (32 dans les Bouches-du-Rhône, 22 dans les Alpes-Maritimes, 18 dans le Var). Si des tendances régionales cohérentes apparaissent, les contre-exemples observés encouragent un changement de focus.

L'espace francilien s'impose comme un observatoire privilégié de cette souscription, et ce, même si une première cartographie simplement régionale n'est pas particulièrement révélatrice (16 donateurs en Seineet-Marne, 18 dans le Val-d'Oise, 27 dans les Yvelines et 28 en Essonne). La projection communale des dons à l'échelle de la première couronne parisienne confirme, elle, en revanche, la marque toute politique du geste des souscripteurs et témoigne de la persistance - « survivance »- du tissu associatif et des réseaux constitutifs du système politico-territorial de la «banlieue rouge ${ }^{67}$. Si des donateurs sont recensés dans 20 communes différentes dans le Val-de-Marne, 21 de la Seine-Saint-Denis et 26 des Hauts-de-Seine, onze d'entre elles concentrent $45 \%$ des dons. Ces onze communes en vue courent sur un arc, au nord de la capitale, de Nanterre à l'Ouest à Montreuil à l'est, de manière discontinue, en passant par Colombes et Argenteuil, et au sud de Malakoff et Montrouge à Champigny-sur-Marne, en passant par Gentilly, Villejuif, Vitry-sur-Seine et Ivry-sur-Seine. Au moins 15 personnes souscrivent à Montreuil, 9 à Ivry-sur-Seine, 8 à Nanterre, 7 à Villejuif, dans des villes constitutives de l'ancien phénomène, politique, culturel, urbain, du « communisme municipal »; une dizaine dans les villes perdues seulement tardivement par le PCF (comme Colombes et Argenteuil en 2001). Largement en déshérence de 1988 et à 2004 de manière progressive - ce qui s'explique notamment par le déclin électoral du

(67) Philippe SUBRA, « Île-de-France : la fin de la banlieue rouge », Hérodote, Paris, Édition la Découverte, 2004/2, n ${ }^{\circ} 13$, p.14-27. 
$\mathrm{PCF}^{68}$-, ce système de la banlieue rouge transparaît pourtant clairement, en filigrane, de cette géographie des dons. Une manifestation évidente validée par les courriers de certains des souscripteurs et révélant le sursaut, ou la nouvelle dynamique durable, d'une gauche non-socialiste ; et attesté depuis par les résultats obtenus par le candidat du Front de Gauche lors du premier tour des élections présidentielles de 2012 (Jean-Luc Mélenchon obtient 24,3\% sur la circonscription de Montreuil, 21,7\% sur celle d'Ivrysur-Seine et du Kremlin Bicêtre, doublant ainsi son score national, mais sur une ligne politique en rupture avec celles d'André Lajoinie, de Robert Hue et Marie-Georges Buffet, et aux côtés d'un candidat socialiste bien plus fortement plébiscité encore). Cette tendance générale d'un don davantage marqué à gauche se confirme elle aussi à l'échelle strictement parisienne. La répartition des dons montre très clairement que davantage de souscripteurs se mobilisent dans les arrondissements du nord, de l'est et du sud de la capitale - et jusque dans le $\mathrm{XV}^{\mathrm{e}}$ - que dans ceux du centre et de l'ouest. Les $\mathrm{I}^{\mathrm{e}}, \mathrm{II}^{\mathrm{e}}, \mathrm{III}^{\mathrm{e}}$ et $\mathrm{XVI}^{\mathrm{e}}$ arrondissements comptent respectivement deux, un, quatre et six donateurs quand ceux des $\mathrm{XIX}^{\mathrm{e}}, \mathrm{XX}^{\mathrm{e}}$ et $\mathrm{XIII}{ }^{\mathrm{e}}$ en dénombrent, eux, dix-huit, vingt-quatre et trente-six ; et la carte des dons de se calquer alors quasi parfaitement sur celles des résultats électoraux enregistrés au printemps 2012 (au premier tour des élections présidentielles, le candidat du Front de Gauche fait ses meilleurs scores, de $15,8 \%$ à 16,8 \%, sur les $6^{\text {ème, }}$ $15^{\text {ème }}, 16^{\text {ème }}$ et $17^{\text {ème }}$ circonscriptions recouvrant assez largement les XIX ${ }^{\mathrm{e}}$ et $\mathrm{XX}^{\mathrm{e}}$ arrondissements, et François Hollande obtient, lui, ensuite de 67,7\% à $72,1 \%$ dans les mêmes et dépasse les $60 \%$ dans le XIII ${ }^{\mathrm{e}}$, au second tour). Faute de dons en très grand nombre, une territorialisation socio-politique aussi nette est impossible à envisager dans d'autres métropoles régionales ; et ce, même si les donateurs de Lyon et de sa proche agglomération (28 sur les 30 Rhodaniens recensés) sont davantage issus des arrondissements $\left(\mathrm{III}^{\mathrm{e}}, \mathrm{IV}^{\mathrm{e}}, \mathrm{VI}^{\mathrm{e}}\right.$ et $\left.\mathrm{IX}^{\mathrm{e}}\right)$ ou des communes voisines du Centre-nord et de l'Est (Villeurbanne), où les candidats de Gauche ont réalisé leurs meilleurs scores lors du scrutin présidentiel (sur les $2^{\text {ème }}, 3^{\text {ème }}$ et $6^{\text {ème }}$ circonscriptions) que de ceux de l'arc Ouest. Au-delà des sensibilités évidentes révélées par cette spatialisation des dons et les motivations exprimées des donateurs, leur dissémination aux quatre coins du pays montre combien en quelques semaines seulement, l'appel prend bien une véritable ampleur nationale. 


\section{Épilogue}

Beaucoup de préparation et d'improvisation, beaucoup de coopération et d'assemblage de compétences ont été nécessaires pour offrir à ceux des souscripteurs qui voulaient et pouvaient se déplacer une fête qui soit à la hauteur de nos remerciements. Le 5 novembre 2011 à Ivry-sur-Seine, dans une belle salle de l'Espace Robespierre prêtée par la municipalité et Pierre Gosnat, député maire, décorée d'expositions patiemment conservées depuis l'époque du Bicentenaire et d'une sélection des mots des souscripteurs, nous avons pu recevoir quelques quatre cents personnes, rendre hommage aux société sœurs et amies, présenter les manuscrits tels que les ateliers des Archives nationales avaient su les photographier en détail, projeter le beau film d'Hervé Pernot sur Robespierre, en débattre fraternellement et finalement remettre le chèque de la souscription à Bruno Galland, directeur scientifique du site de Paris des Archives nationales. Tous les amis, dont notre active porte-parole à l'Assemblée nationale, Catherine Lemorton, et son pendant au Sénat, Jack Ralite, étaient présents. Après toutes ces présentations, vœux et discours, un pot fraternel a ouvert des échanges amicaux, permettant de revenir sur cette occasion exceptionnelle, sur cette première fois où une souscription publique a été organisée pour sauver des $\operatorname{archives}^{69}$ et tenter de mettre en échec ceux qui en font désormais un objet de spéculation financière. Quelques semaines plus tard, à l'Hôtel de Soubise et sur l'invitation des Archives nationales et du ministère de la Culture et de la Communication, ce sont en ces termes que Serge Aberdam et Pierre Serna se félicitent une nouvelle fois de la préservation des manuscrits de Robespierre, dont quelques lettres choisies sont présentées au public.

$\mathrm{Au}$ fond, dans tous ces moments, il a autant été question de biens communs, de patrimoine commun que de la mémoire de la Révolution française ou de celle de Robespierre. Mais dans un autre sens, sur un mode plus discret, l'argument le plus fort peut-être dans les discussions

(69) Cette initiative de la Société des études robespierristes, avec le soutien de l'Institut d'histoire de la Révolution française et de multiples associations amies, a déjà fait date. Nombreux sont les articles, dans la presse écrite notamment, à faire référence à cette démarche citoyenne et à cette conjugaison des forces pour sauvegarder un patrimoine commun de première valeur. Début septembre 2012, des manuscrits inédits d'Émile du Châtelet et de Voltaire refont surface dans des conditions voisines de celles des papiers Robespierre et Lebas. Ils étaient conservés depuis 1892 dans une maison près de Rosnay-L'Hôpital, dans l'Aube, et le restèrent jusqu'en 2010 ; ils sont promis aux enchères chez Christie's à Paris, le 29 octobre suivant. Preuve que les sommes atteintes en mai 2011 ont malheureusement fait jurisprudence, les lots sont estimés à 1 million d'€. Andrew Brown lance alors un appel en faveur de leur sauvegarde et initie une souscription nationale sur le modèle de celle lancée par la Société des études robespierristes. À la date du 19 septembre, quelque 938 personnes avaient déjà répondu à l'appel aux dons. 
était l'existence, avec les deux lots de papiers mis en vente, d'un véritable monument de mémoire, celui d'une militance, celle de la famille Lebas, cachant dès 1794 à ses risques et périls les manuscrits et les conservant dans le plus profond secret tout au long des $\mathrm{XIX}^{\mathrm{e}}$ et $\mathrm{XX}^{\mathrm{e}}$ siècles. Et c'est bien peut-être aussi cette continuité-là qui a tant ému ceux qui savent ce que résister veut dire.

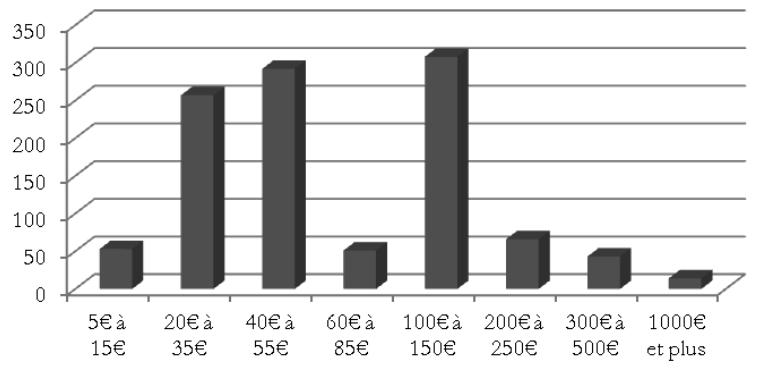

Figure 1. Valeurs des dons enregistrés pour la souscription.

Sources. Archives SER, lettres des souscripteurs.



Figure 2. Part des dons enregistrés dans la souscription. 




Figure 3. Pyramide des âges des souscripteurs (essai de projection graphique).

Sources. Archives SER, lettres des souscripteurs.



Figure 4. Répartition des donateurs par région.

Sources. Archives SER, adresses postales des souscripteurs. 


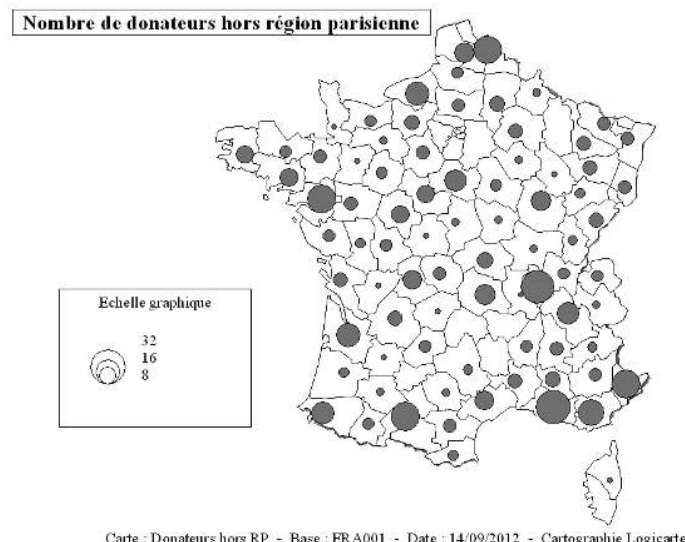

Figure 5. Nombre de donateurs, hors région parisienne.

Sources. Archives SER, adresses postales des souscripteurs.

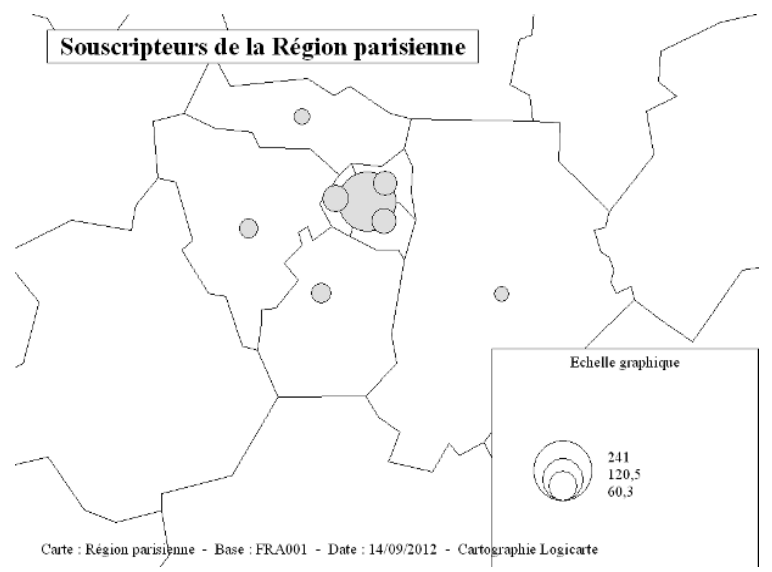

Figure 6. Nombre de donateurs de la région parisienne.

Sources. Archives SER, adresses postales des souscripteurs. 




Figure 7. Nombre de donateurs à Paris.

Sources. Archives SER, adresses postales des souscripteurs.

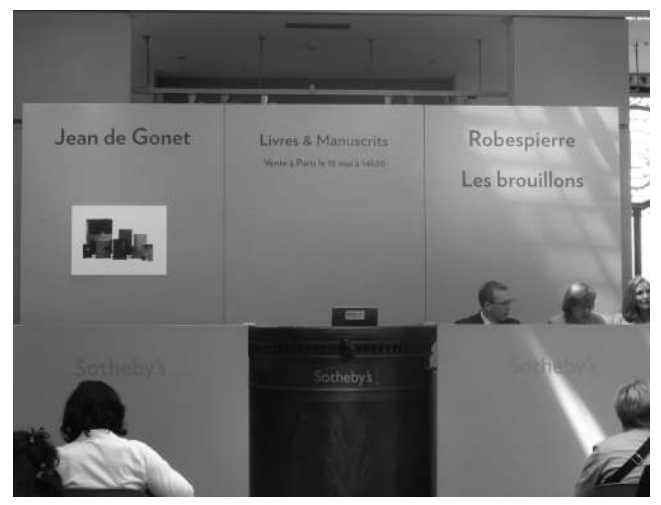

Figure 8. Salle des ventes, manuscrits de Robespierre.

Paris, Sotheby's, le 19 mai 2011. 



Figure 9. Réception et vérification des chèques de souscription.

Paris, Bibliothèque de l'Institut d'histoire de la Révolution française, le 14 juin 2011.

De gauche à droite : Serge Aberdam (secrétaire général), Cyril Triolaire (trésorier), Michel Biard (président) et Jean-Paul Rothiot (responsable des publications).



Figure 10. Remise du chèque de souscription (1).

Ivry-sur-Seine, le 5 novembre 2011.

Cyril Triolaire et Bruno Galland (directeur scientifique du site de Paris des Archives nationales). 




Figure 11. Remise du chèque de souscription (2).

Ivry-sur-Seine, le 5 novembre 2011.

De gauche à droite : Serge Aberdam, Jack Ralite (sénateur), Pierre Gosnat (député-maire d'Ivry-sur-Seine), Catherine Lemorton (députée), Philippe Bourdin (ancien président de la SER) et Bruno Galland.

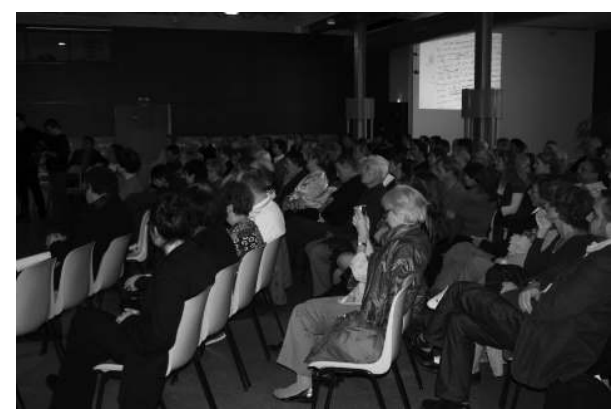

Figure 12. Souscripteurs présents à l'occasion de la fête fraternelle en l'honneur de la sauvegarde des manuscrits de Robespierre.

Ivry-sur-Seine, le 5 novembre 2011

Serge ABERDAM

INRA

63 Boulevard de Brandebourg

94205 Ivry cedex serge.aberdam@ivry.inra.fr

Cyril Triolaire

CHEC, Université Blaise Pascal (Clermont 2)

CHEC, MSH, 4 rue Ledru, 63000 Clermont-Ferrand cyril.trio@wanadoo.fr 